\title{
Analytical Protocols for Separation and Electron Microscopy of Nanoparticles Interacting with Bacterial Cells
}

\author{
Cláudia Sousa, ${ }^{\dagger}$ Diana Sequeira, ${ }^{\dagger}$ Yury V. Kolen'ko, ${ }^{\ddagger}$ Inês Mendes Pinto, ${ }^{\ddagger}$ and Dmitri Y. Petrovykh ${ }^{*, \neq}$ \\ ${ }^{\dagger}$ Center of Biological Engineering, University of Minho, Braga 4710-057, Portugal \\ ${ }^{\ddagger}$ International Iberian Nanotechnology Laboratory, Braga 4715-330, Portugal
}

Supporting Information

\begin{abstract}
An important step toward understanding interactions between nanoparticles (NPs) and bacteria is the ability to directly observe NPs interacting with bacterial cells. $\mathrm{NP}$-bacteria mixtures typical in nanomedicine, however, are not yet amendable for direct imaging in solution. Instead, evidence of $\mathrm{NP}$-cell interactions must be preserved in derivative (usually dried) samples to be subsequently revealed in high-resolution images, for example, via scanning electron microscopy (SEM). Here, this concept is realized for a mixed suspension of model NPs and Staphylococcus aureus bacteria. First, protocols for analyzing the relative colloidal stabilities of NPs and bacteria are developed and validated based on systematic centrifugation and comparison of colony forming

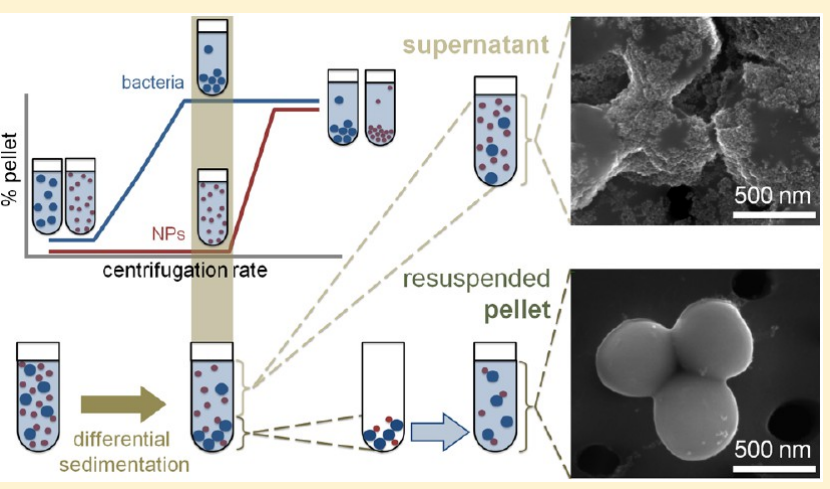
unit (CFU) counting and optical density (OD) measurements. Rate-dependence of centrifugation efficiency for each component suggests differential sedimentation at a specific predicted rate as an effective method for removing free NPs after co-incubation; the remaining fraction comprises bacteria with any associated NPs and can be examined, for example, by SEM, for evidence of NP-bacteria interactions. These analytical protocols, validated by systematic control experiments and high-resolution SEM imaging, should be generally applicable for investigating NPbacteria interactions.
\end{abstract}

$\mathrm{T}$ he unique physicochemical properties of nanoparticles (NPs) are the basis for their potential applications in nanomedicine, whereby NPs interacting with cells provide a means for detecting, monitoring, or controlling cell functions via nonbiological properties of NPs (magnetic, electronic, optical, mechanical). ${ }^{1-3}$ Interactions of NPs with bacterial cells, for example, have been studied for overcoming antibiotic resistance, ${ }^{4,5}$ biosensing, ${ }^{6-9}$ and gene delivery. ${ }^{10}$

Despite the significant progress in characterizing interactions between NPs and bacteria, ${ }^{11-14}$ an important remaining challenge is the lack of a general method that can provide direct, systematic, and quantitative observations of NPs interacting with bacteria. The current approaches tend to primarily focus on biological or physical aspects of such samples. A commonly used biological assay, for example, tests the antimicrobial effect of NPs by evaluating the bacterial viability after exposure to NPs. ${ }^{15,16}$ Physical aspects of NPbacteria interactions tend to be analyzed by high-resolution imaging techniques, such as electron, ${ }^{8,9,11,12,14}$ atomic force, ${ }^{17}$ or Raman ${ }^{13}$ microscopies, which can provide direct and quantitative information about NPs and cells (such as proximity, penetration, aggregation) but typically not directly in solution environments where NPs and bacteria interact. Artifacts introduced during preparation for imaging, therefore, can obscure evidence of NP-bacteria interactions.
If a protocol can be developed to minimize samplepreparation artifacts, scanning electron microscopy (SEM) would offer significant benefits for imaging NPs interacting with bacterial cells. Field-of-view (FOV) dimensions accessible by SEM enable capturing statistically significant numbers of resolved individual cells within a single image [Figures S1-S2 in the Supporting Information (SI) $].{ }^{18,19}$ The high spatial resolution and depth-of-field of SEM also enable simultaneous observation of $\mu \mathrm{m}$-scale objects, such as bacteria, and NPs resolved individually or in aggregates (Figure 1).

The concentrations of cells and NPs dramatically influence the preparation and SEM imaging of NP-bacteria mixtures. Samples with low concentrations of both cells and NPs (Figure le and f) are typically the easiest to image at high resolution; ${ }^{9,20}$ however, isolated individual areas of such samples (e.g., Figure $\mathrm{S} 2 \mathrm{~b}, \mathrm{SI}$ ) may not be representative of the whole and the low concentration of NPs may have limited their opportunity to interact with particular cells in each FOV (Figure 1f). Conversely, having a high concentration of NPs (or cells) in mixed samples increases the possibility of observing artifacts from both preparation and imaging procedures (Figure 1a-

Received: October 13, 2014

Accepted: March 12, 2015

Published: March 12, 2015 

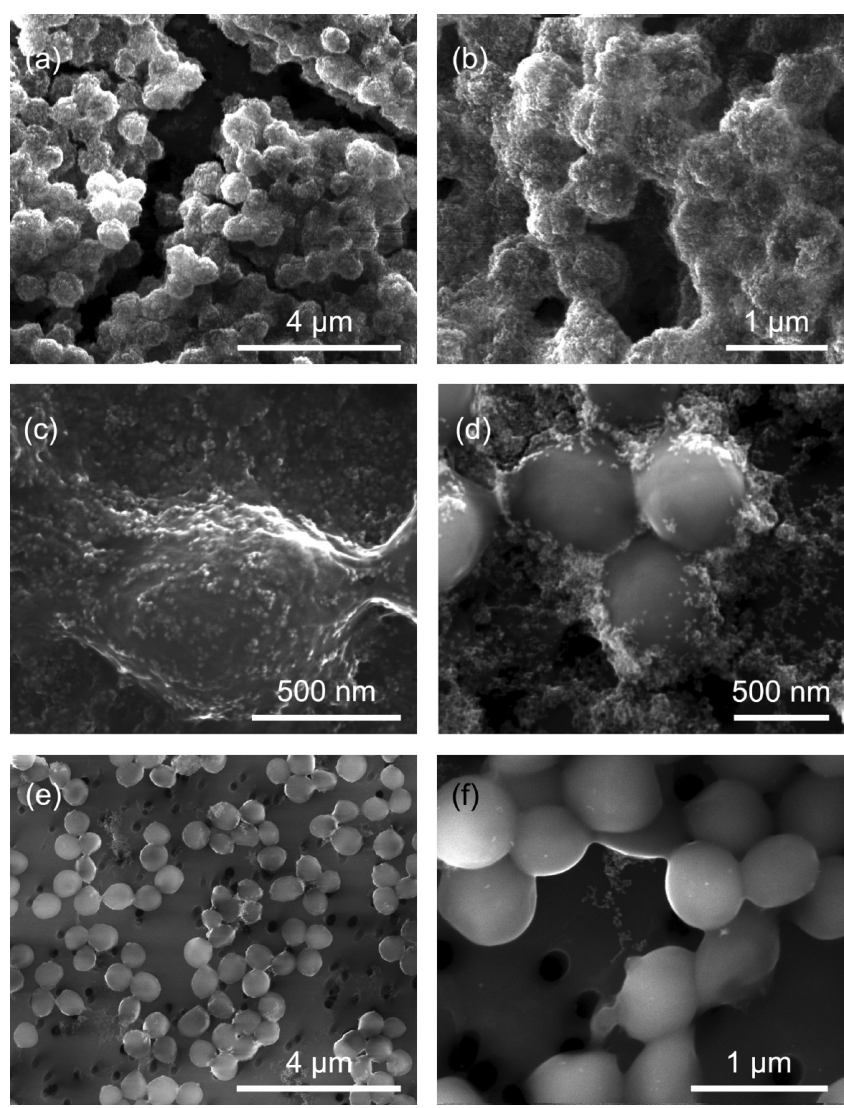

Figure 1. Sample-preparation artifacts observed by SEM for mixed samples of NPs and S. aureus. High concentration of both bacteria and NPs produces samples with several layers of bacteria covered with a rough, dense overlayer of NPs (a, b). Low concentration of bacteria mixed with a high concentration of NPs produces individual bacteria coated with overlayers (c) or aggregates (d) of NPs. Lowering the concentration of NPs results in concentration-limiting their attachment to bacteria (e, f).

d). ${ }^{14,15}$ While it obfuscates evidence of specific NP-cell interactions, coating of cells with a dense layer of NPs is successfully used, for example, in membrane-protein enrichment protocols, which are commonly illustrated with SEM images resembling our Figure $1 \mathrm{a}-\mathrm{c}^{21-24}$ A high concentration of NPs during incubation, therefore, provides a simple general method for ensuring that all the cells had the opportunity to interact with NPs before having been prepared for imaging.

The key to preparing optimal samples of bacteria and any associated NPs for SEM imaging is, therefore, in controlling the NP concentration throughout the process. Specifically, starting with an excess of NPs in the incubation solution produces a mixture of free NPs and NPs associated with bacteria. To avoid artifacts during imaging, free NPs are then removed before drying the sample. Conversely, any NPs that became associated with bacteria during co-incubation may reveal evidence and properties of the underlying NP-bacteria interactions under direct, systematic, and quantitative ex situ analysis.

The importance of ensuring that all the free NPs have been removed after co-incubation depends on the context of an experiment. For example, when verifying high loading of each cell with NPs, ${ }^{21-24}$ including NPs in a form of dense layers (Figure 1a and $b$ ) or aggregates (Figure 1d), even a relatively high fraction (perhaps, as high as $30 \%$ ) of free NPs among all NPs sedimented with cells would not affect the interpretation of results dramatically. The salient common characteristic of such experiments is the relative unimportance of distinguishing between NP-NP and NP-cell interactions. Conversely, when attempting to investigate specificity of NP-cell interactions, ${ }^{8,9}$ any artifacts related to the presence of free NPs need to be carefully minimized, to avoid misinterpreting the apparent results, especially in comparative studies of different NPs, for example. This caveat becomes singularly important when attempting to verify that $\mathrm{NP}$-cell interactions are minimal (or even absent), as would be required in measurements of negative controls in studies of specific NP-cell interactions.

For separating free NPs from a co-incubation mixture, we propose centrifugation because it relies on the colloidal stability of bacteria and NPs; the latter can be controlled via physicochemical properties of NPs and the solvent. ${ }^{25}$ Furthermore, centrifugation is routinely used for separation and concentration of bacteria ${ }^{26,27}$ as well as $\mathrm{NPs}^{28,29}$ or NPloaded fragments of cell membranes. ${ }^{21-24}$ For larger ( 5-15 $\mu \mathrm{m})$ cells, dense coating with NPs can be sufficient to sediment the NP-loaded cells from an NP-cell mixture based on their higher density. ${ }^{21,23,24}$ For mixtures of NPs and submicrometersized bacteria with relatively low NP loading, however, densitybased sedimentation may not be very effective, so more general colloidal stability differences have to be used for differential sedimentation, especially when a nearly complete removal of free NPs, as discussed above, is the goal. Here, we demonstrate a basic example of a systematic methodology for performing a deterministic separation of free NPs from a co-incubation mixture, whereby the initial separate measurements of NPs and cells are used (1) to narrow down the mixed NP-bacteria samples parameter space (which, a priori, is vast, owing to the combinatorics of possible mixtures) and (2) to predict the parameters for separation of mixed NP-bacteria samples. This analytical separation protocol produces samples of bacterial cells and any NPs associated with them that can be prepared for subsequent direct observation by SEM (or by other microscopy or spectroscopy methods $)^{8,13}$ to obtain information about NPbacteria interactions that occurred during their co-incubation.

\section{EXPERIMENTAL SECTION}

Nanoparticles with Organic Coatings. Magnetite NPs were synthesized by coprecipitation (CP) in an automated reactor or by a hydrothermal method (HT), as described previously (SI). ${ }^{25}$ The synthesized NPs were stabilized by oleate (OL) or poly(acrylic acid) (PAA) capping ligands. Tetramethylammonium hydroxide (TMAOH) surfactant was used to transfer hydrophobic as-synthesized OL-coated NPs to aqueous phase. ${ }^{30}$ Hereafter, the NPs are denoted by the capping ligand (PAA or OL) and the synthesis protocol (CP or HT).

Colloidal Stability of NPs. The iron oxide NPs used in this work are superparamagnetic, ${ }^{25}$ so their magnetic properties do not induce colloidal instability. As described in the text and the SI, sedimentation threshold during centrifugation was used to evaluate the colloidal stability of NPs in four aqueous solutions: $\mathrm{NaCl} 0.9 \%, \mathrm{NaCl} 0.45 \%, 0.01 \mathrm{M}$ phosphate buffered saline, $\mathrm{pH}$ 7.2 (PBS $0.01 \mathrm{M}$ ), and distilled water.

Staphylococcus aureus Suspensions. For each experiment, a stock suspension of Staphylococcus aureus (S. aureus) was prepared as described in the SI. The standard protocol involves 3 (washing) steps, during which bacteria are pelleted at $8000 \mathrm{rpm}$ and resuspended by vortexing. After the final washing, pellets were resuspended in $30 \mathrm{~mL}$ of the desired 
aqueous solution and sonicated under conditions that disrupt cell clusters without impairing cell viability. ${ }^{31}$ The suspension was then adjusted to a specific optical density at $640 \mathrm{~nm}$ $\left(\mathrm{OD}_{640}\right)$ for individual experiments.

$S$. aureus Viability. The viability of $S$. aureus cells was evaluated in aqueous solutions of the same composition as those used to test the colloidal stability of NPs. Cell suspensions were prepared and plated as described in the SI, followed by comparing the colony forming unit (CFU) counts. $^{32}$

Sedimentation Efficiency for S. aureus. Sedimentation efficiency for $S$. aureus in water was determined starting with a cellular suspension adjusted to $\mathrm{OD}_{640} \approx 0.6$. Additional measurements at $\mathrm{OD}_{640} \approx 0.1$ and 0.2 are described in the SI. For each value of centrifugation rate (1000-8000 rpm), two separate $5 \mathrm{~mL}$ samples of $S$. aureus suspension were centrifuged for $5 \mathrm{~min}$. After centrifugation, the $\mathrm{OD}_{640}$ was measured for the supernatant, which was aseptically decanted to a new tube and vortexed, and the pellet, which was resuspended in $5 \mathrm{~mL}$ of water, vortexed, and sonicated (as detailed in the SI).

SEM Imaging of S. aureus and NPs. After centrifugation, each sample (supernatant or resuspended pellet) was filtered through a sterile $25 \mathrm{~mm}$-diameter polycarbonate filter with $\sim 0.2 \mu \mathrm{m}$ track-etched pores (Whatman, GE Healthcare Life Sciences) mounted in a funnel assembly connected to a vacuum pump. The material retained on the filter was fixed and dehydrated as described in the SI. Samples were imaged in a Quanta 650 (FEI) field-emission SEM (SI Table S1).

Statistical Analysis. Confidence intervals (CI) of mean values were calculated using the statistical package of Microsoft Excel 2010, assuming the Student's $t$-distribution with $n$ replicates $(n<30)$. The CIs are reported at $95 \%$ confidence level (CL) as mean $\pm t_{0.95 / 2} \cdot s / \sqrt{n}(s=$ standard deviation $)$.

\section{RESULTS AND DISCUSSION}

Our approach to developing and validating a protocol for optimal separation and SEM imaging of nanoparticles interacting with bacteria is summarized in Scheme 1. We begin by establishing the parameter window for differential sedimentation, which we propose to use for removing excess free NPs (Figure 1a-d) after co-incubation of NPs and bacteria. Multiple options for choosing NPs and/or solution conditions (Table 1) make direct sedimentation measurements for all possible combinations of parameters impractical. Instead, we first evaluate the colloidal stability of NPs and bacteria separately in systematic centrifugation experiments that are designed to identify a range of rpm values where free NPs remain in the supernatant while bacteria sediment (Scheme 1a).

We take advantage of the relatively simple colloidal stability measurements for NPs to isolate the combination of NP and solution properties that is likely to produce efficient removal of free NPs from co-incubation mixtures (Table 1). Colloidal stability of $S$. aureus bacteria is then quantified in the solution identified from NP experiments and after validating the measurement methods. Finally, we compare the observed sedimentation thresholds with the pattern in Scheme 1a, and perform the separation (Scheme 1b) followed by SEM imaging.

Colloidal Stability of NPs. A common benefit of using NPs in biomedical applications is the ability to optimize the NP coating and solution conditions, for example, to maximize the desired NP-cell interactions. The same two parameters (NP coating and solution conditions) affect the colloidal stability
Scheme 1. Differential Sedimentation: Colloidal Stability Analysis (a) Predicts the Rate for Efficient Separation (b)
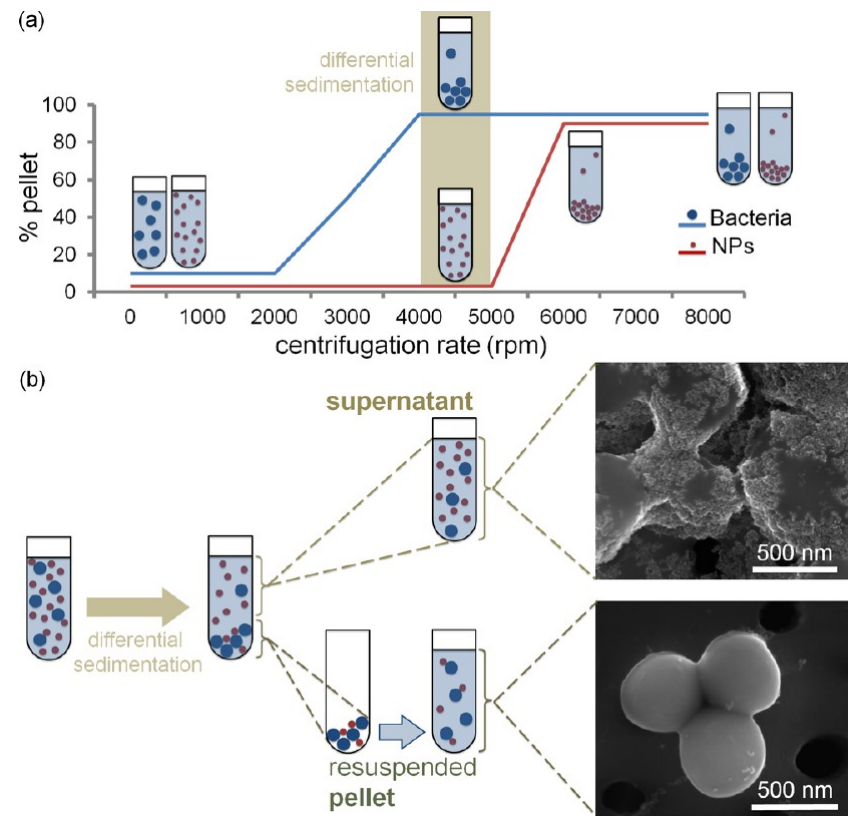

Table 1. Colloidal Stability of NPs under Centrifugation

\begin{tabular}{|c|c|c|c|c|c|}
\hline \multirow[b]{4}{*}{ solutions } & \multicolumn{2}{|c|}{ coprecipitation } & \multicolumn{3}{|c|}{ hydrothermal } \\
\hline & \multicolumn{3}{|c|}{ poly(acrylic acid) } & \multicolumn{2}{|c|}{ oleic acid + TMAOH } \\
\hline & PAA-CP1 & PAA-CP2 & PAA-HT & OL-HT1 & OL-HT2 \\
\hline & \multicolumn{5}{|c|}{$\begin{array}{l}\text { the lowest centrifugation rate (rpm) at which sedimentation } \\
\text { was observed }\end{array}$} \\
\hline $\mathrm{NaCl} 0.9 \%$ & 1000 & 2000 & 5500 & 1000 & 1000 \\
\hline $\mathrm{NaCl} 0.45 \%$ & 1000 & 3000 & 4000 & 1000 & 1000 \\
\hline PBS $0.01 \mathrm{M}$ & 1000 & 3500 & 3000 & 1000 & 1000 \\
\hline water & 3500 & 3500 & 3000 & 2000 & 6500 \\
\hline
\end{tabular}

and thus their combination can be optimized for removing free NPs from mixtures via differential sedimentation (Scheme 1).

To illustrate our methodology, we consider a series of five NPs in which similar superparamagnetic iron oxide cores are coated with different organic shells (Table 1 and the SI). ${ }^{25}$ The solution options for NP-cell systems are commonly dictated by the biological components, such as any antibodies or enzymes involved in biofunctionalization of NPs and biorecognition as well as the cells themselves; the need to maintain viability of $S$. aureus, for example, determined our solution choices (Table 1).

Having the solution conditions constrained by the biological component limits applicability of the otherwise common practice whereby changes in solution conditions (e.g., $\mathrm{pH}$, ionic strength, or temperature) are used to evaluate the colloidal stability of NPs. ${ }^{25}$ Serendipitously, the intended use of centrifugation suggests the use of sedimentation thresholds for comparing colloidal stabilities of NPs in one or more solutions.

For each combination of NP type and aqueous solution, sedimentation threshold, i.e., the lowest centrifugation rate at which NPs sedimented, was established (Table 1). For each NP-solution pair, three separate aliquots were tested starting from $1000 \mathrm{rpm}$; if sedimentation was not observed, another three aliquots were tested at a higher rate (in $500 \mathrm{rpm}$ increments). In all cases, we observed abrupt sedimentation, i.e., at a given rate, NP suspensions either remained stable or changed color and formed a pellet (appearance similar to SI 
Figure S3c). Accordingly, thus determined sedimentation threshold could be used as a quantitative index of relative colloidal stability.

The highest colloidal stability was observed for OL-HT2 (TMAOH-stabilized) in distilled water (Table 1), which remained in suspension up to $6000 \mathrm{rpm}$, suggesting the use of water as a solvent and OL-HT2 as model NPs for demonstrating the differential sedimentation approach (Scheme 1).

S. aureus Viability. While water has been identified above as a good solvent for OL-HT2 NPs, unlike saline solutions, distilled water is not commonly used to maintain Staphylococcus viability. ${ }^{33}$ Keeping in mind the ultimate objectives of studying interactions of NPs with live bacteria, we started by evaluating the viability of $S$. aureus in water.

In a common viability assay for bacterial cells, diluted aliquots of the bacterial suspension are placed onto a solid growth medium and incubated overnight. The number of visible colonies that form after the incubation is counted; the resulting number of colony forming units (CFUs) can be used to evaluate bacterial viability, e.g., by comparing with CFU counts from reference experiments. ${ }^{32,34,35}$ As detailed in the SI, we compared the CFU counts for $S$. aureus after incubation in water to CFU counts reached after incubation in three other solvents: PBS $0.01 \mathrm{M}$ and $\mathrm{NaCl} 0.9 \%$, which are commonly used with $S$. aureus, and $\mathrm{NaCl} 0.45 \%$; the latter was chosen as an intermediate value between water and $\mathrm{NaCl} 0.9 \%$, to check for any trends in viability with salt concentration.

In three assays performed on different days, we started from the same initial $S$. aureus concentration (adjusted to $\mathrm{OD}_{640} \approx$ 0.1 ) and found countable plates (with 6-9 replicates) in the same dilution, therefore, the CFU counts could be compared directly (Figure 2, SI Figure S4), rather than via conversion to

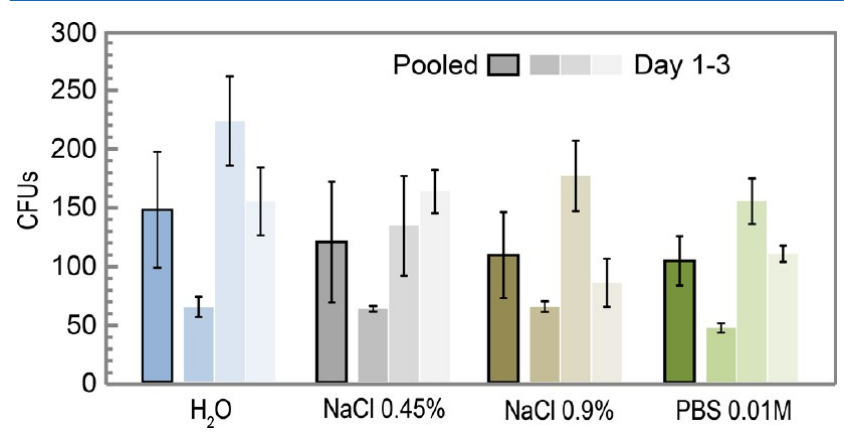

Figure 2. Viability of $S$. aureus after $1 \mathrm{~h}$ incubation in water or in salt solutions. For each solution, CFU counts (in the same dilution) are shown for 3 separate assays (lighter shading) and their pooled mean value (outline, dark shading). Error bars indicate 95\% CIs, as detailed in the SI.

representation as nominal $\mathrm{CFU} / \mathrm{mL}$ values commonly used in microbiology. ${ }^{35,36} \mathrm{CFU}$ counts for plates prepared after incubation for $1 \mathrm{~h}$ indicate some variability among results obtained for different solutions or on different days (Figure 2), but the mean values (dark shading in Figure 2) are not significantly different, based on the pooled 95\% CIs. Mean CFU counts also were not significantly different at 95\% CL among plates prepared after incubation for $24 \mathrm{~h}: 134 \pm 57,97$ $\pm 18,111 \pm 20$, and $109 \pm 16$ for water, $\mathrm{NaCl} 0.45 \%, \mathrm{NaCl}$ $0.9 \%$, and PBS $0.01 \mathrm{M}$, respectively. Comparing these CFU counts, we can reach two conclusions about $S$. aureus viability. First, there is no significant difference in $S$. aureus viability after either 1 or $24 \mathrm{~h}$ incubation in the four solutions that we have examined. Second, there is no evidence of significant death or growth of $S$. aureus when incubated for up to $24 \mathrm{~h}$ in all four solutions. Accordingly, even though a previous study indicated a difference in viability of $S$. aureus in water vs PBS over several weeks, ${ }^{35}$ our measurements validate the use of distilled water in experiments with $S$. aureus incubation times $\leq 24 \mathrm{~h}$.

Our statistical analysis, detailed in the SI, provides additional important indications. The variability of our results is not unusual for CFU counting data, which typically are reported in $\log _{10}$ plots and analyzed in terms of order-of-magnitude differences. $^{36,38}$ Reproducibility (95\% CI) of our same-day replicates-a common quality-assurance indicator ${ }^{37,38}$-is in $\pm 10 \%$ to $\pm 20 \%$ range, which is in line with best-practice measurements ${ }^{32,38}$ and thus would not provide, by itself, a compelling justification for additional measurements after completing the first set of assays. Repeating the assays on different days, however, revealed that the uncertainty of CFU measurements likely includes a significant systematic component, in addition to the expected random variation. We emphasize that the level of variability in our data would be inconsequential in a typical microbiology context, ${ }^{36}$ e.g., establishing a minimum bactericidal concentration, but it must be considered when using CFU counting as a bacterial viability or concentration measurement.

Quantification of $S$. aureus Concentration. Having verified $S$. aureus viability in water, we can proceed to measure the colloidal stability of these suspensions. Unlike for the case of NPs (Table 1), however, we do not have the advantage of abrupt visible sedimentation for bacterial suspensions. Accordingly, to evaluate the sedimentation efficiency as a gradual function of the centrifugation rate, we need to identify a method suitable for measuring bacterial concentrations.

Turbidity or optical density (OD) is commonly measured for suspensions of bacterial cells. ${ }^{39,40}$ At bacterial concentrations relevant for surface attachment, however, reliably measuring $\mathrm{OD} \approx 0.1$ can be a challenge, because blank buffers commonly have OD around $0.03-0.05$. CFU counting can reach a lower detection limit than does OD but is more labor-intensive and time-consuming than OD measurements. Another important consideration is obtaining practically useful uncertainty (e.g., of $<50 \%$ ) for sedimentation efficiency values, which involve ratios of two measurements; therefore, the uncertainty of each measurement of a bacterial concentration should be $<20 \%$.

To test the resolution of OD and CFU methods, three solutions were prepared to differ by $20 \%$ (based on dilution factors) in bacterial concentration. For a method with resolution sufficient to distinguish these $20 \%$ differences in concentration, we would expect the measured means to trend and to be significantly different across the concentration series.

From the comparison in Figure 3 (and data in SI Table S2), the resolution of the CFU measurements clearly is not sufficient to distinguish the samples in this range of bacterial concentrations. For OD measurements, the means trend as expected and are significantly different at 95\% CL for the two larger values, indicating that the resolution of OD measurements is sufficient for quantifying concentration ratios for samples with $O D \geq 0.1$.

Sedimentation Efficiency for S. aureus. The appropriate initial concentration for the samples is largely determined by our interest in following sedimentation efficiency as a function of the centrifugation rate, whether that dependence is abrupt (as we observe for NPs) or gradual. For the latter case, the 


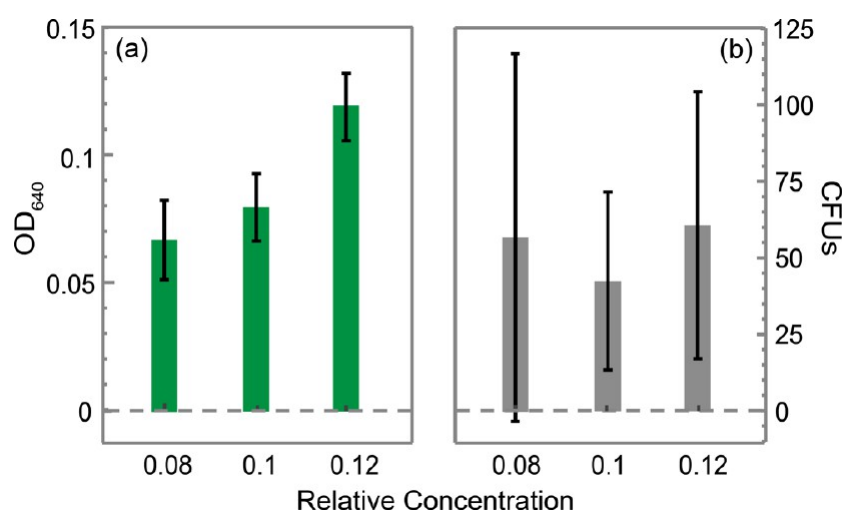

Figure 3. Resolution of cell concentration measurements. $\mathrm{OD}_{640}$ values (a) and CFU counts (b) are compared for $S$. aureus suspensions prepared as a dilution series with $20 \%$ increments. Error bars indicate $95 \%$ CIs $(n=4)$; data summarized in SI Table S2.

smallest detectable pellet dictates the choice of the initial concentration. Measuring a pellet formed by as little as $20 \%$ of the sample is practically useful; taking into account the $\mathrm{OD} \geq$ 0.1 limit established above, we arrive to $\mathrm{OD}_{640}$ in $0.5-0.6$ range as an appropriate initial concentration.

The data from systematic measurements presented in Figure 4 (and detailed in SI Table S3) validate our approach to

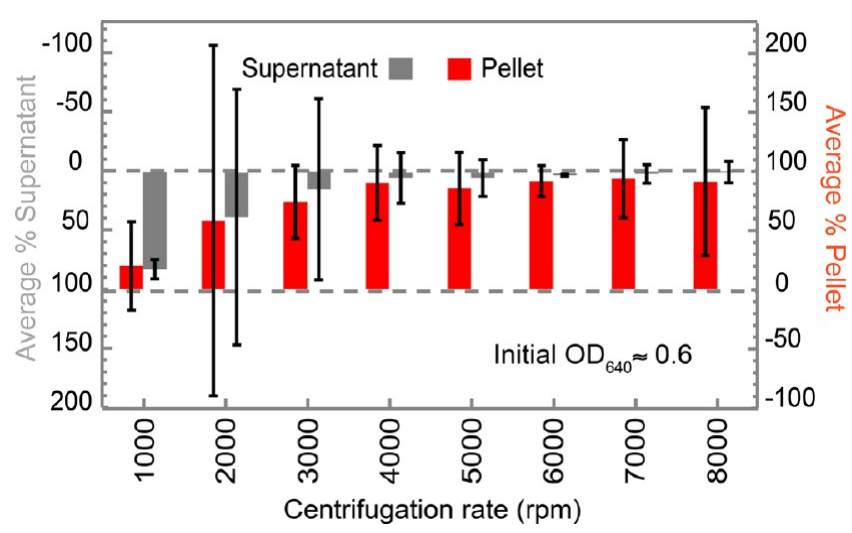

Figure 4. Sedimentation efficiency for $S$. aureus. For each rpm value, $\mathrm{OD}_{640}$ was measured for a separate aliquot of the initial suspension. The measured $\mathrm{OD}_{640}$ values (SI Table S3) for both supernatant (gray bars, left axis) and resuspended pellet (red bars, right axis) are represented relative to the initial $\mathrm{OD}_{640} \approx 0.6$; error bars indicate $95 \%$ CIs, $n=2$ from two separate experiments.

measuring the sedimentation efficiency for $S$. aureus. In particular, our choices of OD measurements and of the initial concentration have enabled us to observe the gradual increase in sedimentation efficiency from 1000 to $4000 \mathrm{rpm}$. The sedimentation efficiency clearly plateaus above $4000 \mathrm{rpm}$, indicating a parameter window between 4000 and $6000 \mathrm{rpm}$ for attempting the differential sedimentation of bacteria and free NPs (Scheme 1, Table 1). We repeated the measurements at 1000,4000 , and $6000 \mathrm{rpm}$ (in triplicate for each rpm value) to confirm the presence of the efficiency plateau in the 4000$6000 \mathrm{rpm}$ range (SI Figure S5) and the reproducibility of our strategy.

We note that the sensitivity limit of the OD measurements effectively restricts reliable measurements of the sedimentation efficiency to relatively high initial concentrations of bacteria. The OD value of $\approx 0.6$ in Figure 4 is actually above the concentration optimal for incubating with an excess of NPs and for subsequently depositing the entire sample on the nanoporous support for SEM analysis. Our attempts to measure the sedimentation efficiency starting from OD values of $0.1-0.2$ (SI Figure S6) were inconclusive because they produced much larger uncertainties, in agreement with results discussed in the previous two sections. We believe, however, that for our objective of producing a representative sample of bacteria and any NPs that directly interacted with them during co-incubation, assuring $>90 \%$ sedimentation efficiency is desirable but not indispensable. For example, if the practical efficiency will enable us to recover about $50 \%$ of the bacteria from the mixed co-incubation sample, that fraction is likely to be representative of the population that we intend to analyze because a $50 \%$ fraction will not be dominated exclusively by mutant strains or other similarly low probability artifacts.

Removal of Free NPs after Co-incubation with $S$. aureus. Having performed colloidal stability measurements on S. aureus and NPs separately (Scheme 1a), we proceeded to the final step of the approach outlined in Scheme $1 \mathrm{~b}$, whereby the results of those separate measurements are combined to define the parameter window for differential sedimentation of the mixed co-incubation sample of $S$. aureus and NPs. We started from a co-incubation sample prepared with an excess of OLHT2 NPs (as described in the SI) to maximize the possibility for NP-cell interactions. The mixture was incubated for $1 \mathrm{~h}$, homogenized by vortexing, and centrifuged for $5 \mathrm{~min}$ at 4000 rpm, i.e., the value at which we expected to produce differential sedimentation of bacteria (along with any NPs that had become associated with them during co-incubation), while separating free NPs into the supernatant (Scheme 1). Both the supernatant and resuspended pellet were then prepared for SEM analysis by filtering onto nanoporous membranes followed by fixation and dehydration. SEM images of the material retained on the nanoporous membranes are presented in Figures 5 and 6, and SI Figure S7.

An early indication of successfully retaining the free NPs in the supernatant was its brown color. After preparation for SEM, the supernatant sample shown at different magnifications in the panels of Figure 5 and SI Figure S7 is clearly dominated by structures formed by the drying of free NPs, as evidenced, for example, by observing similarly large/thick aggregates of NPs forming both on top of cells and on the supporting membrane without any cells in direct proximity. Cracks in the NP overlayer, e.g., visible around the "(a)" marker in Figure 5a, are also likely drying artifacts, supporting the above interpretation. Figure 5 thus clearly illustrates how difficult (if not impossible) it would be to find indications of NP-cell interactions without removing all free NPs after the co-incubation step.

Inferences from SEM Images for NP-Cell Interactions in Solution. In contrast to artifact-dominated images in Figure 5 , features likely produced due to $\mathrm{NP}$-cell interactions in solution are visible in SEM images that show the resuspended pellet sample in Figure 6, at magnifications similar to those in Figure 5. The NPs bound to the bacteria in Figure 6 can be presumed to have attached during co-incubation, as the bulk of the unbound NPs clearly have been removed from this sample during centrifugation. A close inspection of the supporting membrane reveals that the removal of NPs not bound to the cells has been remarkably successful. Observing only a trivial number of free NPs, we conclude that any artifacts associated with them can be considered negligible in these images. 

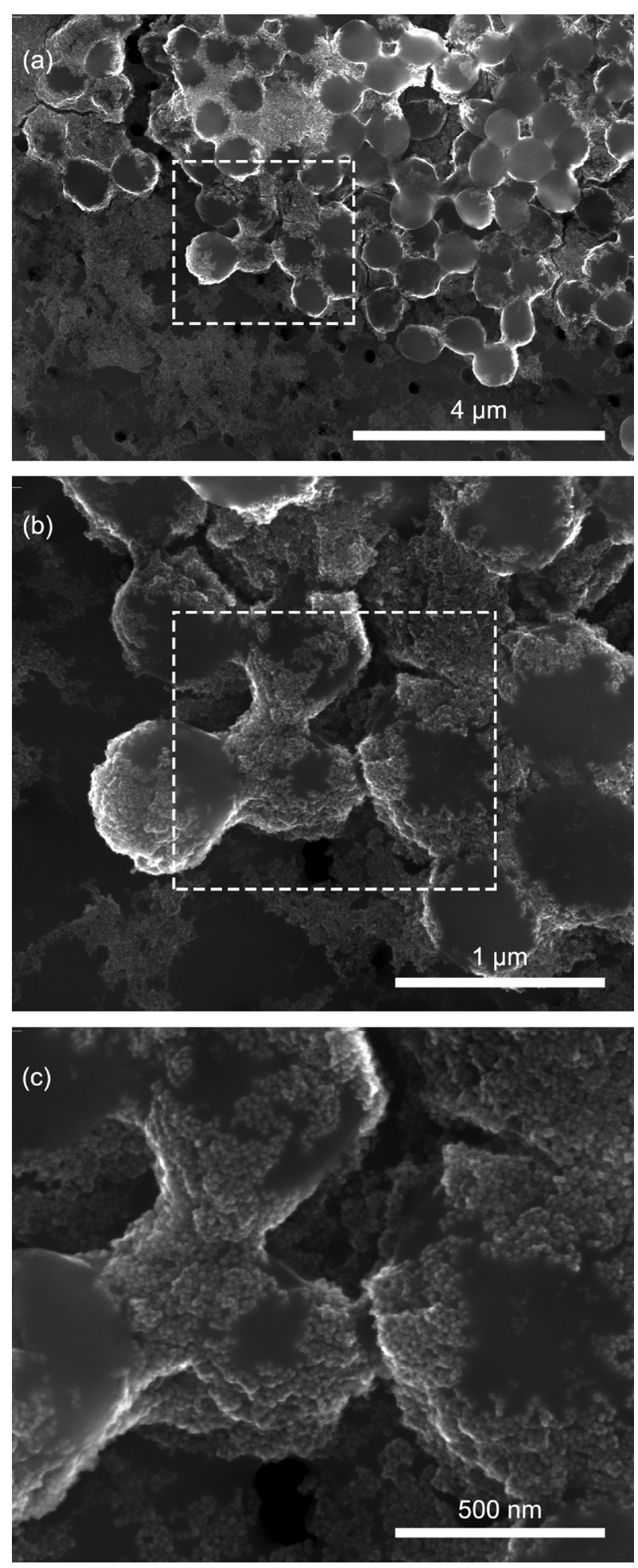

Figure 5. SEM images of the supernatant after differential sedimentation of an NP-cell mixture. Following Scheme 1b, free OL-HT2 NPs remained in supernatant at $4000 \mathrm{rpm}$. NPs appear as resolved bright objects $(\sim 20 \mathrm{~nm})$ or as aggregates; S. aureus cells appear as spherical objects $(\sim 700 \mathrm{~nm})$. Panels $\mathrm{b}$ and $\mathrm{c}$ correspond to the outlined regions in panels $a$ and $b$, respectively.

Multiple features in panels of Figure 6 support the possibility of making inferences about $\mathrm{NP}$-cell interactions, based on samples prepared using our protocols. Large differences in NP loading of adjacent cells (Figure 6e), apparent clustering of NPs around some of the cell adherence regions (Figure 6b), and the prevalence of individual NPs or apparently loose NP aggregates (indicated by observing discrete NPs or linear rather than 3D aggregates on cell surfaces) are all examples of qualitative features that could readily form during the co-incubation stage due to NP-cell interactions, to which the discrete character and localization of NP features may be attributed. Conversely, formation of such discrete and localized features during the stochastic processes, such as filtering and drying, would be much more difficult to rationalize.

Considering the colloidal stability of NPs (Table 1) provides additional insight for interpreting the NP aggregates observed in Figures 5 and 6. Abruptness of sedimentation observed for all our NPs strongly indicates that formation of even small NP aggregates rapidly destabilizes the suspension. Accordingly, any NP aggregates formed during the co-incubation step would be pelleted during centrifugation. In contrast, any NPs retained in the supernatant could not have been substantially aggregated during co-incubation. The absence of large NP aggregates in Figure 6 then supports the assumption that during coincubation the individual NPs dominated the NP-cell interactions; conversely, the presence of aggregates in Figure 5 must be related to preparation artifacts.

We note that even though Figure 5 suggests that the sedimentation efficiency for $S$. aureus cells in this experiment did not reach the $90 \%$ range achieved in Figure 4, it clearly remained above 50\%; therefore, as discussed before, our preparation protocol has successfully produced samples amendable for elucidating the existence and properties of solution-phase NP-cell interactions. While our model NPs had not been functionalized to produce specific attachment to $S$. aureus bacteria, SEM images in Figure 6 illustrate the feasibility of future investigations of NPs attached to bacteria via putative specific interactions. ${ }^{8,9}$ Furthermore, any bacteria that internalize some of the NPs will be also naturally represented in NPcell samples prepared using our protocol. Our model NPs are not designed to promote specific internalization and, indeed, are not internalized in any significant numbers, as confirmed by EDX measurements (SI Figures S8-S9). For appropriately functionalized NPs, however, systematic analysis of their internalization by bacteria will be an important area of future analytical developments ${ }^{41,42}$ that can take advantage of our protocols.

\section{CONCLUSIONS}

We developed and validated a protocol for producing samples that are amendable for elucidating from SEM images the existence and properties of solution-phase NP-cell interactions. The proposed elements of the workflow, including the initial separate investigations of colloidal stabilities for NPs and cells, have been illustrated by successfully separating, via differential sedimentation, and preparing for SEM characterization samples of NPs attached to $S$. aureus bacteria.

We used sedimentation thresholds as quantitative indices to compare relative colloidal stability of NPs under centrifugation. We found $O D$ values to be suitable for quantifying sedimentation of $S$. aureus cells under centrifugation after systematically comparing bacterial enumeration via $\mathrm{OD}$ and CFU methods. Our insights and methodology will be important for future analytical developments for NP-bacteria samples.

While illustrated using a specific model system, all the steps of our protocol can be readily adapted to NPs or bacterial cells 

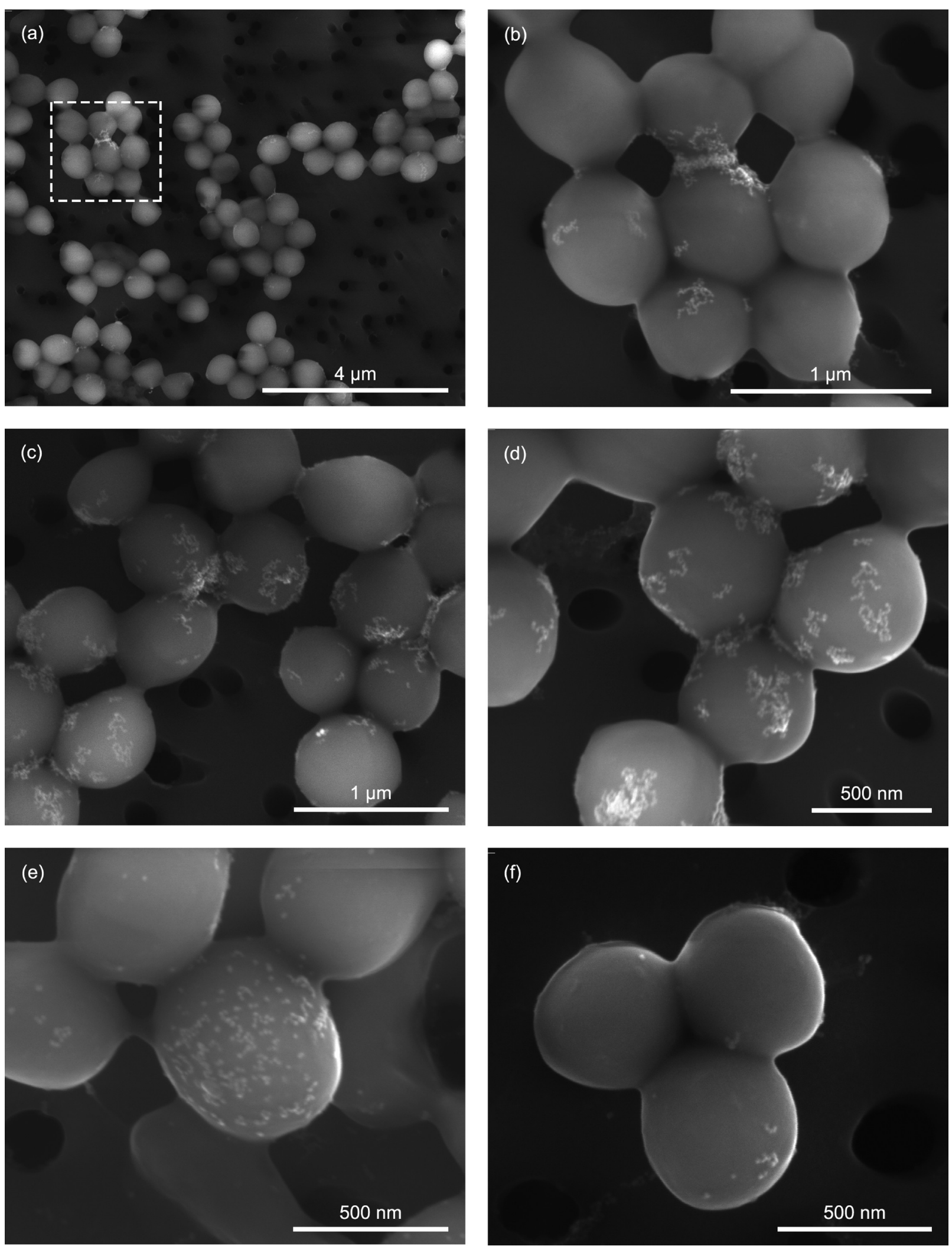

Figure 6. SEM images of the resuspended pellet after differential sedimentation of an NP-cell mixture. After centrifugation at $4000 \mathrm{rpm}$, no free NPs were expected in the pellet (Scheme 1). S. aureus cells appear as spherical objects $(\sim 700 \mathrm{~nm})$; numerous bright NPs $(\sim 20 \mathrm{~nm})$ and loose NP aggregates are observed on cells but not on porous membrane support; panel b corresponds to the outlined region in panel a.

that have somewhat different properties, particularly because most biomedical applications involve cells and NPs that are colloidally stable under a common set of conditions and offer possibilities for controlling the relative stabilities of the different components. Our approach thus should be generally applicable 
for preparing samples that will enable direct and quantitative investigations of interactions between NPs and bacteria.

\section{ASSOCIATED CONTENT}

\section{S Supporting Information}

Materials and methods, SEM images, SEM settings, EDX spectra, detailed comparison of $\mathrm{OD}_{640}$ and $\mathrm{CFU}$ data, statistical analysis of viability data, and sedimentation efficiency. This material is available free of charge via the Internet at http:// pubs.acs.org.

\section{AUTHOR INFORMATION}

\section{Corresponding Author}

*E-mail: dmitri.petrovykh@inl.int.

\section{Notes}

The authors declare no competing financial interest.

\section{ACKNOWLEDGMENTS}

C.S. acknowledges financial support from the following sources: grant SFRH/BPD/47693/2008 from the Portuguese Foundation for Science and Technology (FCT); FCT Strategic Project PEst-OE/EQB/LA0023/2013; project "BioHealth-Biotechnology and Bioengineering approaches to improve health quality", Ref. NORTE-07-0124-FEDER-000027, cofunded by the Programa Operacional Regional do Norte (ON.2-O Novo Norte), QREN, FEDER; project "Consolidating Research Expertise and Resources on Cellular and Molecular Biotechnology at CEB/IBB", ref. FCOMP-01-0124-FEDER027462. The authors thank Dr. Leonard Deepak Francis (INL) and Keriya Mam (FEI) for helpful discussions and assistance provided for SEM and EDX analysis.

\section{REFERENCES}

(1) Huang, X.; El Sayed, M. A. J. Adv. Res. 2010, 1, 13-28.

(2) Wang, W.; Li, S.; Mair, L.; Ahmed, S.; Huang, T. J.; Mallouk, T. E. Angew. Chem. 2014, 126, 3265-3268.

(3) Chen, J.-S. Front. Mater. 2014, 1, 1-2.

(4) Hajipour, M. J.; Fromm, K. M.; Ashkarran, A. A.; Jimenez de Aberasturi, D.; de Larramendi, I. R.; Rojo, T.; Serpooshan, V.; Parak, W. J.; Mahmoudi, M. Trends Biotechnol. 2012, 30, 499-511.

(5) Seil, J. T.; Webster, T. J. Int. J. Nanomedicine 2012, 7, 2767-2781.

(6) Tallury, P.; Malhotra, A.; Byrne, L. M.; Santra, S. Adv. Drug Delivery Rev. 2010, 62, 424-437.

(7) Syed, M. A. Biosens. Bioelectron. 2014, 51, 391-400.

(8) Wang, J.; Gao, J.; Liu, D.; Han, D.; Wang, Z. Nanoscale 2012, 4, 451-454.

(9) Chang, Y.-C.; Yang, C.-Y.; Sun, R.-L.; Cheng, Y.-F.; Kao, W.-C.; Yang, P.-C. Sci. Rep. 2013, 3, 1863.

(10) Lee, Y. H.; Wu, B.; Zhuang, W. Q.; Chen, D. R.; Tang, Y. J. J. Microbiol. Methods 2011, 84, 228-233.

(11) Chwalibog, A.; Sawosz, E.; Hotowy, A.; Szeliga, J.; Mitura, S.; Mitura, K.; Grodzik, M.; Orlowski, P.; Sokolowska, A. Int. J. Nanomed. 2010, 5, 1085-1094.

(12) Hayden, S. C.; Zhao, G.; Saha, K.; Phillips, R. L.; Li, X.; Miranda, O. R.; Rotello, V. M.; El-Sayed, M. A.; Schmidt-Krey, I.; Bunz, U. H. J. Am. Chem. Soc. 2012, 134, 6920-6923.

(13) Vishnupriya, S.; Chaudhari, K.; Jagannathan, R; Pradeep, T. Part. Part. Syst. Charact. 2013, 30, 1056-1062.

(14) Rodrigues, D.; Bañobre-López, M.; Espiña, B.; Rivas, J.; Azeredo, J. Biofouling 2013, 29, 1225-1232.

(15) Sondi, I.; Salopek-Sondi, B. J. Colloid Interface Sci. 2004, 275, $177-182$.

(16) Chatterjee, S.; Bandyopadhyay, A.; Sarkar, K. J. Nanobiotechnology 2011, 9, 34.
(17) Gammoudi, I.; Faye, N. R.; Moroté, F.; Moynet, D.; GraubyHeywang, C.; Cohen-Bouhacina, T. Int. J. Chem. Mater. Sci. Eng. 2013, $79,607-613$.

(18) Afrikian, E. G.; Julian, G.; Bulla, L. Appl. Microbiol. 1973, 26, 934-937.

(19) Bergmans, L.; Moisiadis, P.; Van Meerbeek, B.; Quirynen, M.; Lambrechts, P. Int. Endod. J. 2005, 38, 775-788.

(20) Pagnout, C.; Jomini, S.; Dadhwal, M.; Caillet, C.; Thomas, F.; Bauda, P. Colloids Surf., B 2012, 92, 315-321.

(21) Chaney, L. K.; Jacobson, B. S. J. Biol. Chem. 1983, 258, 1006210072.

(22) Stolz, D. B.; Jacobson, B. S. J. Cell Sci. 1992, 103, 39-51.

(23) Choksawangkarn, W.; Kim, S. K.; Cannon, J. R.; Edwards, N. J.; Lee, S. B.; Fenselau, C. J. Proteome Res. 2013, 12, 1134-1141.

(24) Kim, S. K.; Rose, R.; Choksawangkarn, W.; Graham, L.; Hu, J.; Fenselau, C.; Lee, S. B. J. Nanopart. Res. 2013, 15, 2133.

(25) Kolen'ko, Y. V.; Bañobre-López, M.; Rodríguez-Abreu, C.; Carbó-Argibay, E.; Sailsman, A.; Piñeiro-Redondo, Y.; Cerqueira, M. F.; Petrovykh, D. Y.; Kovnir, K.; Lebedev, O. I.; Rivas, J. J. Phys. Chem. C 2014, 118, 8691-8701.

(26) Maggi, C.; Lepore, A.; Solari, J.; Rizzo, A.; Di Leonardo, R. Soft Matter 2013, 9, 10885-10890.

(27) Fukushima, H.; Katsube, K.; Hata, Y.; Kishi, R.; Fujiwara, S. Appl. Environ. Microbiol. 2007, 73, 92-100.

(28) Kowalczyk, B.; Lagzi, I.; Grzybowski, B. A. Curr. Opin. Colloid Interface Sci. 2011, 16, 135-148.

(29) Akbulut, O.; Mace, C. R.; Martinez, R. V.; Kumar, A. A.; Nie, Z.; Patton, M. R.; Whitesides, G. Nano Lett. 2012, 12, 4060-4064.

(30) Salgueiriño-Maceira, V.; Correa-Duarte, M. A.; Farle, M. Small 2005, 1, 1073-1076.

(31) Freitas, A. I.; Vasconcelos, C.; Vilanova, M.; Cerca, N. J. Basic Microbiol. 2014, 54, 750-757.

(32) Jett, B. D.; Hatter, K. L.; Huycke, M. M.; Gilmore, M. S. Biotechniques 1997, 23, 648-650.

(33) Chapman, J. H. J. Bacteriol. 1945, 50, 201-203.

(34) Greenberg, A., Clesceri, L., Eaton, A., Eds. In Standard Methods for the Examination of Water and Wastewater, 18th ed.; American Public Health Association: Washington, D.C., 1992.

(35) Liao, C.-H.; Shollenberger, L. M. Lett. Appl. Microbiol. 2003, 37, 45-50.

(36) Pankey, G. A.; Sabath, L. D. Clin. Infect. Dis. 2004, 38, 864-870.

(37) Barbosa, H. R.; Rodrigues, M. F. A.; Campos, C. C.; Chaves, M. E.; Nunes, I.; Juliano, Y.; Novo, N. F. J. Microbiol. Methods 1995, 22, $39-50$.

(38) Sutton, S. J. Validation Technol. 2011, 17 (3), 42-46.

(39) Sutton, S. J. Validation Technol. 2011, 17 (1), 46-49.

(40) Koch, A. L. Anal. Biochem. 1970, 38, 252-255.

(41) Kumar, A.; Pandey, A. K.; Singh, S. S.; Shanker, R.; Dhawan, A. Cytometry 2011, 79A, 707-712.

(42) Yao, Z.; Carballido-López, R. Annu. Rev. Microbiol. 2014, 68, 459-76. 